\title{
CONFLICTOS COGNITIVOS, EXPERIMENTOS CUALITATIVOS Y ACTIVIDADES DIDÁCTICAS*
}

\author{
VILLANI, A. ${ }^{1}$ y ORQUIZA DE CARVALHO, L. ${ }^{2}$ \\ Instituto de Física. Universidade de São Paulo. Caixa Postal 20516. São Paulo. SP. Brasil \\ Facultade de Engenharia. Universidade Paulista. Campus Ilha Solteira. SP. Brasil.
}

\section{SUMMARY}

The article reIates the results of a qualitative research with secondary school students on the cognitive conflicts about central collisions in mechanics. We put our attention to the interviewer's mediation in the origins, developments and conclusions of the conflicts. We analyze the main results of the process and sketch some pedagogical consequences.

\section{INTRODUCCIÓN}

Las investigaciones sobre las representaciones mentales de los estudiantes cuentan ya con veinte años de historia (Driver, 1973; Malgrange et al., 1973), durante la cual las posturas de los investigadores han ido evolucionando. Los esfuerzos recientes están dirigidos hacia la elaboración de estrategias eficientes que favorezcan el cambio conceptual (Nussbaum y Novick, 1982; Roweli y Dawson, 1985; Grimellini et al., 1989; Dreyfus et al., 1990; Duschl y Gitomer, 1991; Brown y Clement, 1992). En muchos casos, se considera que los conflictos cognitivos son la puetta privilegiada (Scott et al., 1992) para aumentar el conocimiento científico de los estudiantes y hacerlo más estable. En particular, la potencialidad de los experimentos y su capacidad de contribuir a los cambios deseados han constituido un importante punto de discusión (Nachtingal, 1992). Algunos investigadores han subrayado tanto la necesidad de producir situaciones experimentales que posibiliten contrastar las ideas como la posibilidad de suscitar los correspondientes conflictos cognitivos; otros, en cambio, se han mostrado críticos y escépticos al respecto (Hodson, 1988; Nersessian, 1989), destacando el rol fundamental que tiene el modo de ver de los estudiantes. Recientemente Chinn y Brewer (1993) han discutido exhaustivamente el papel de los datos anómalos en la adquisición del conocimiento científico, mostrando la similitud entre el modo de proceder de ios científicos y de los estudiantes. Es interesante en particular su descripción de las diver- sas posibilidades de aceptación o rechazo de los datos experimentales por parte de los estudiantes.

En este trabajo analizaremos la producción de conflictos cognitivos, principalmente mediante el uso de experiencias sencillas, y cómo surge del análisis de una parte de los datos de una investigación realizada en la Universidad de S. Paulo con algunos estudiantes de escuela media superior.

Comenzaremos nuestra exposición comentando algunas características de la investigación; luego describiremos y clasificaremos los conflictos cognitivos generados durante las entrevistas a los estudiantes realizadas por uno de los autores (L.O.C.) y finalmente analizaremos las estrategias utilizadas por la entrevistadora para desarrollar los conflictos. Conciuiremos delineando algunas consecuencias pedagógicas de los resultados obtenidos.

\section{LA INVESTIGACIÓN}

La investigación consistió en una serie de entrevistas (siete u ocho) sobre los choques en mecánica con cada uno de los estudiantes participantes. La parte que se analiza aquí corresponde a las dos primeras entrevistas, 
en las cuales se desarrolló una discusión sobre algunos experimentos sencillos relativos a choques centrales. Se trata de doce entrevistas, de 60 a 90 minutos de duración, registradas parte en audio (las cuatro primeras) y parte en vídeo (las ocho restantes ${ }^{3}$ ) en las cuales la entrevistadora interactuó en modos diversos con el estudiante entrevistado con la finalidad de aclarar sus ideas, de desarrollar y, posiblemente, de iniciar una confrontación con la visión disciplinar.

En el apéndice A se hace una descripción más detallada de las actividades desarrolladas por la entrevistadora y de los objetivos correspondientes.

La población objeto de estudio está compuesta por seis estudiantes de la escuela media superior, cuyas edades están comprendidas entre los quince y los veintidós años: tres mujeres (CE, THA y DA) y tres varones (PA, NA y FE), elegidos entre los conocidos de la entrevistadora según un criterio de comodidad y en modo de garantizar la continuidad del trabajo, ya que se trataba de realizar una serie completa de entrevistas sobre la física. Desde el punto de vista de las capacidades intelectuales, dos de los estudiantes pueden considerarse brillantes (NA y THA), tres con capacidad media (CE, DA y PA) y uno con muchas dificultades (FE).

El análisis y comparación del comportamiento de los estudiantes, que será objeto de este trabajo, se vio faci- litado por la relativa homogeneidad del guión de las entrevistas analizadas, durante las cuales se usaron los mismos dispositivos experimentales y se realizaron aproximadamente los mismos experimentos. Éstos se describen en el apéndice $B$.

\section{LOS CONFLICTOS COGNITIVOS DE LOS ESTUDIANTES}

La característica principal de la dinámica de las entrevistas fue la producción y desarrollo de conflictos cognitivos; es decir, de perturbaciones intelectuales, capaces de provocar insatisfacción en el estudiante con respecto a sus propios conocimientos. La clasificación exhaustiva de los distintos tipos de conflictos (Dykstra, 1992) está más allá de las expectativas de este trabajo, en el que analizaremos sólo algunos aspectos y sus respectivas consecuencias desde el punto de vista didáctico. Centraremos nuestra atención en el origen, dinámica y conclusión de los conflictos; elaboraremos algunas categorías significativas que aparecen en el esquema 1 en la parte inferior, las cuales nos permitirán analizar de manera crítica las intervenciones didácticas de la entrevistadora y definir objetivos específicos para desarrollar una estrategia de enseñanza dirigida a producir el cambio conceptual a través del conflicto cognitivo.

Esquema 1. Dimensiones y categorías de los conflictos cognitivos.

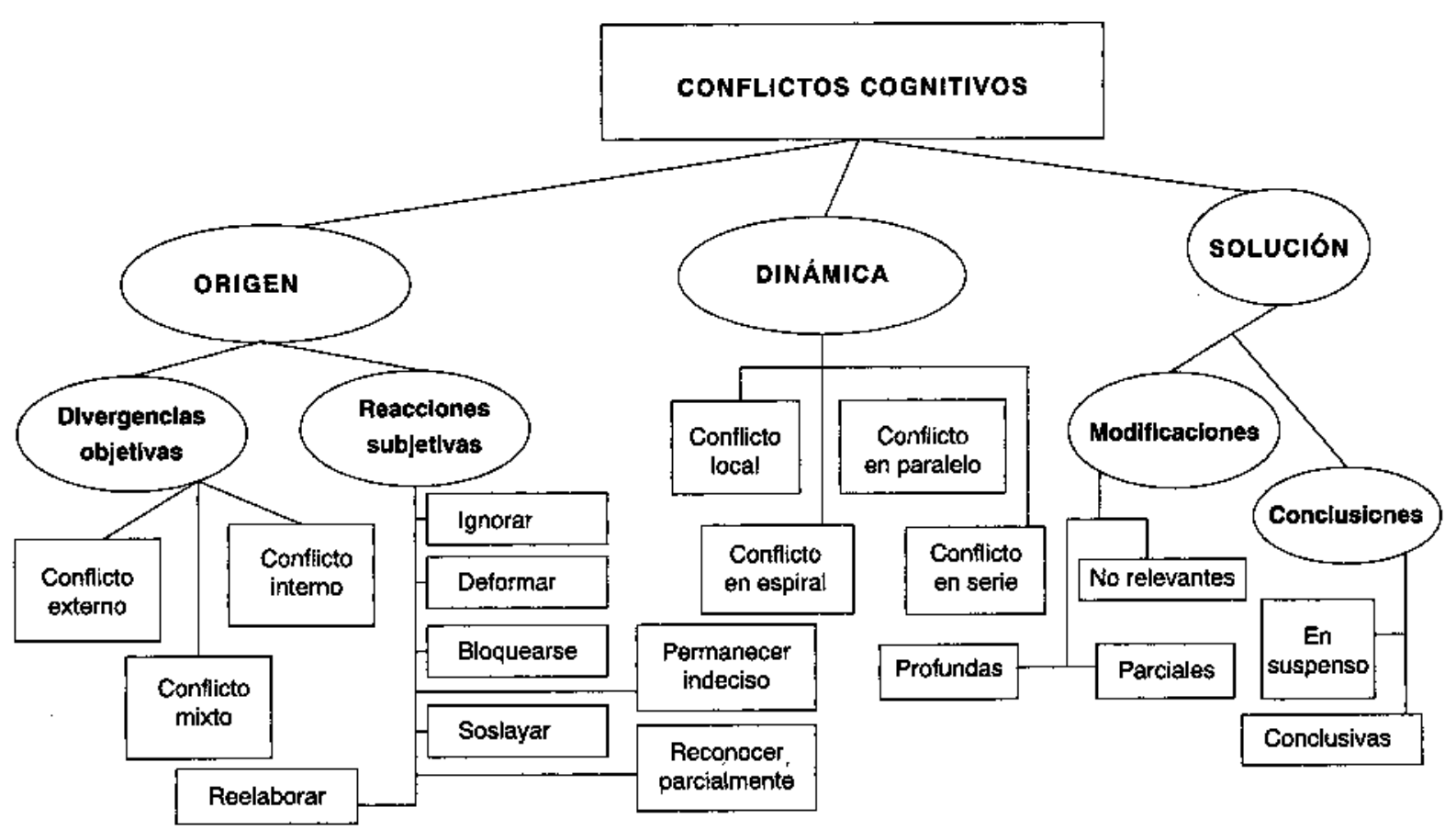




\section{Los orígenes de los conflictos}

Nos centraremos en dos aspectos de las carencias cognitivas, que están en la base de los conflictos cognitivos: las divergencias objetivas entre los elementos cogniti. vos, individualizadas mediante el análisis detallado de las entrevistas y su contexto, y la consecuente reacción subjetiva del estudiante, interpretada a partir de los indicios por él mismo aportados.

\section{A. Las divergencias objetivas}

Un dato que nos ha impresionado durante el análisis de las entrevistas fue la variedad de elementos que constituyen los conflictos: previsiones, observaciones, explicaciones, aspiraciones cognitivas, sentimientos del estu. diante, resultados experimentales, sugerencias de la entrevistadora, informaciones escolares y lecturas didácticas. En función de la presencia de estos elementos, hemos definido tres categorías: conflictos externos, in " ternos y mixtos.

- Conflictos externos. Denominamos externos a aquellos conflictos caracterizados por una divergencia entre los modos de ver del estudiante y los elementos externos a él, como el resultado de un experimento, la observación de la entrevistadora o bien la frase de un libro de texto.

El conflicto externo más simple es aquel constituido por la divergencia entre las ideas del estudiante sobre los experimentos y el resultado de los mismos; tal divergencia puede manifestarse tanto en las previsiones persona. les, como en las explicaciones. Una situación de conflicto que aparece muchas veces es la previsión de la detencion del proyectil en p-4 o en c-5 y la explicación de la diferencia entre deslizarse y rodar mediante la diferencia de velocidad del proyectil.

Otro tipo de divergencia de origen externo se produce cuando las sugerencias o los puntos de vista de la entrevistadora contrastan con las ideas de los estudian* tes. Dos ejemplos particularmente signifícativos son: la sugerencia, dada a todos los estudiantes, de la deformación (invisible) de las bolitas de acero, que contrasta con la observación espontánea de los estudiantes; y la solicitud de concentrar sistemáticamente la atención en el momento del choque en contraste con la atención dirigida espontáneamente a la fuerza ejercida sobre el proyectil y al correspondiente efecto sobre el blanco (Villani y Pacca, 1990; Grimellini et al., 1993).

En algunos casos, la divergencia entre el modo de mirar de la entrevistadora y el modo de ver del estudiante asumía aspectos altamente sofisticados que implicaban valores epistemológicos como la capacidad de percibir analogías. Por ejemplo, durante la segunda entrevista con NA, la entrevistadora orientó sus preguntas de modo que el estudiante percibiera que el experimento c- 8 con las bolitas de acero podía ser interpretado como la unión del experimento $\mathrm{c}-8$ totalmente plástico (imaginado con bolitas de plastilina) con el experimento $t-2$ (conl los carritos) en el cual se producía una explosión.
También pudimos constatar conflictos entre las ideas del estudiante y las formuladas en sus libros de texto, revelados por las preguntas formuladas a la entrevistadora. Por ejemplo, FE, al comentar la frase de un libro de texto que había leído durante el intervalo entre la primera y la segunda entrevista, manifestó duđas sobre la duración de la interacción, que no lograba comparar con otros intervalos temporales familiares; durante la discusión sucesiva se vio claramente que la divergencia básica entre sus ideas y las del libro correspondía a la concepción de interacción entre los dos objetos que colisionaban.

- Conflictos internos. Hemos denominado conflictos internos a aquéllos caracterizados por una divergencia entre elementos cognitivos internos del estudiante, como sus percepciones, sus ideas, sus exigencias epistemoló. gicas o cognitivas (Hewson, 1985) y, en general, su ecología conceptual (Posner et al., 1982; Strike y Posner, 1992).

Un caso interesante, que fue observado varias veces, es el de la divergencia entre una convicción espontánea y un conocimiento escolar. THA, por ejemplo, afirmó que el movimiento ascendente de una moneda era mantenido por una fuerza y, simultáneamente, que existía una relación, precedentemente aprendida en la escuela, entre fuerza y aceleración.

Otro caso interesante es el de la divergencia entre las premisas y las conclusiones de un razonamiento. FE se encontró varias veces en esta situación porque en su discurso se intercalaban fácilmente ideas implícitas ajenas al punto en discusión.

Otras veces la divergencia se producía entre las conclusiones, a menudo parciales, elaboradas por el estudiante y su exigencia de una explicación general. Una situación de este tipo se produjo con CE, que buscaba una variable única responsable del movimiento del proyectil después del choque y arribó a la conclusión de que una vez era la velocidad, otra vez exa la masa y otra, la fuerza del choque.

Una situación análoga se producía con mucha frecuencia, cuando la entrevistadora pedía al estudiante que explicitara conceptos fundamentales, citados precedentemente por él y éste enunciaba frases a veces incoherentes o confusas; casi siempre el estudiante terminaba confesando que no sabía enunciar dichos conceptos, revelando implícitamente un contraste entre su explici. tación y sus exigencias de claridad y precisión en relación con los conceptos científicos.

Finalmente, debemos reconocer que durante las en. trevistas hemos hallado también un tipo diferente de conflicto interno, de carácter principalmente emotivo, en el que el estudiante dudaba entre implicarse desde el punto de vista intelectual y, eventualmente, estar en desacuerdo con la entrevistadora o bien, simplemente, tratar de adaptarse a sus exigencias sin exponerse (Baid y Mitchell, 1986; Villani, 1992). 
-Conflictos mixtos. En algunos casos los conflictos presentaban una estructura compleja que incluía varias divergencias simultáneas: cuando éstas se referían tanto a elementos internos como a elementos externos, el conflicto se consideraba mixto. Por ejemplo, en el extenso conflicto experimentado por NA en la primera entrevista, entraban en juego las divergencias entre las dos leyes de Newton, la equivalencia implícita entre velocidad y aceleración y los resultados de $\mathrm{c}-5$ (Apéndice $\mathrm{C}$ ).

\section{B. Las reacciones de los estudiantes}

Cuando el estudiante se encontraba frente a uno o más conflictos objetivos ${ }^{4}$, podía pasar lo siguiente:

-No tener conciencia en modo alguno de las divergencias. El caso más frecuente se verificaba cuando en el discurso del estudiante afloraban varias divergencias objetivas y éste, como máximo, lograba individualizar una de ellas. La tarea de hacer aflorar las restantes era delegada invariablemente a la entrevistadora, como en el caso de la explicación del choque en el péndulo, en el que la separación entre las variables significativas y la gra. vedad y el rozamiento requirió siempre de varias intervenciones sucesivas.

-Negar, deformar o, por lo menos, minimizar los elementos divergentes. Por ejemplo, CE continuaba afir* mando, aun después de la demostración experimental, que en el experimento p-2 las bolitas de acero después del choque frontal, prácticamente elástico, no alcanzaban la altura inicial, y FE sostenía que en c-2, después del choque, el proyectil se detenía inmediatamente. Otras veces, la idea sugerida por la entrevistadora no era considerada o, en caso de insistencia, era descartada como poco plausible, como ocurrió con CE respecto a la deformación del acero durante el choque.

- Soslayar el problema, discutiendo sin individualizar los elementos esenciales (conflicto incipiente). Por ejemplo, al comienzo de la primera entrevista, FE no lograba comprender la importancia de concentrar la atención en el momento del choque y de explicar los resultados mediante relaciones precisas entre las variables que caracterizaban las bolitas interaccionantes; por esto continuaba respondiendo en términos de análisis perceptivo global.

-Bloquearse cognitivamente, es decir, no poder continuar el diálogo con la entrevistadora. Una reacción de este tipo se producía conjuntamente con los conflictos emotivos: en el caso de PA, la presencia de esta perturbación, probablemente debida a factores extraños a la entrevista, se mantuvo bastante tiempo comprometiendo gran parte de la primera entrevista; en cambio, para THA fue un paréntesis de breve duración, rápidamente superado, debido a una toma de conciencia dramática, de sus ideas espontáneas relativas a fuerza y movimiento.

-Reconocer sólo parcialmente la divergencia conside. rándola como una excepción. Por ejemplo, en el caso de PA, habiendo previsto, en base a su modelo de acción y reacción, que en p-2 las dos bolitas de acero se deten- drían y después de haber observado el rebote, en c-8 repitió la misma previsión, utilizando el mismo modelo de acción y reacción. En otros casos la previsión exa considerada provisotia y era sustituida fácilmente por otra más adecuada, frecuentemente adhoc, sin dar lugar a ningún otro tipo de conflicto. Por ejemplo, NA, frente a la previsión incorrecta de un movimiento de todas las bolitas del blanco en $\mathrm{c}-7$, basada probablemente en la idea de un blanco único, después de algunas observaciones reconoció que el fenómeno era regulado por un principio de simetría y que, en consecuencia, se habrían de mover tantas bolitas del blanco como las que componían el proyectil.

-Reconocer la divergencia permaneciendo indeciso sin hacer una elección. Una situación muy interesante se verificaba cuando la divergencia entre previsión y observación se debía a una teoría aprendida explícitamente en la escuela. En un primer momento el conflicto se producía entre los valores cognitivos atribuidos a las dos fuentes de conocimiento: el estudiante quedaba perplejo, sin saber si descartar la teoría o el experimento. A veces se esforzaba por reconciliar ambas ideas, como sucedió con $\mathrm{CE}$, cuando quería salvar Ia jđea, aprendida en la escuela, de que en el choque existe disipación y el resultado experimental de p-1, que mostraba una oscilación residual del proyectil. Se observó una situación análoga cuando el estudiante, frente al compromiso de dar una justificación, reconoció tener las ideas poco claras. Sobre todo cuando estaba en juego la definición de un concepto importante, apenas se le proporcionaba la ocasión, pedía aclaraciones a la entrevistadora o bien buscaba en los libros una respuesta más adecuada. Esto sucedió con CE quien, al comienzo de la segunda entre vista preguntó qué era el impulso, y con $F E$ que, en el intervalo entre la primera y la segunda entrevistas, buscó información sobre lo que sucedía durante el cho" que, lo cual había sido incapaz de describir precedentemente.

-Reconocer la divergencia y reelaborar sus ideas. Tal reelaboración podía asumir una profundidad y una amplitud variables. En general un resultado experimental disonante constituía una fuente de perturbación signifi. cativa cuando la previsión era fruto de una teoría perso. nal. Un ejemplo representativo es la previsión de THA sobre la detención del proyectil en p-4, debida a su convicción de que ta transmisión de la energía se produ. cía siempre en la dirección del movimiento: la observa. ción del rebote del proyectil representó para ella una sorpresa tal que la impuisó no sólo a redimensionar su idea inicial sino también a buscar otro tipo de explicación. Una situación semejante se verificaba cuando el estudiante consideraba plausibles o, al menos, pertinen. tes las sugerencias de la entrevistadora que contrastaban con sus ideas espontáneas. Por ejemplo, la sugerencia de la existencia de una deformación no visible del acero, mediante la cual la entrevistadora trataba de ayudar a los estudiantes a resolver el problema de la elasticidad de los choques, indujo a $\mathrm{FE}$ a concluir que el acero debía sufrir «una deformación parcial, similar a la elasticidad parcial de la madera". THA, en cambio, después de quedar inicialmente algo perpleja, comprendió la potencia 
unificadora de la idea y la aceptó, llegando incluso a utilizarla en la deducción de consecuencias interesantes.

\section{La dinámica de los conflictos}

Otro aspecto que ha llamado nuestra atención fue la evolución de los conflictos. Hemos definido cuatro tipos fundamentales: local, en paralelo, en serie y en espiral.

-El conflicto local se desarrolla y termina por libre iniciativa del estudiante. Un ejempio es el caso de CE que, después de haber analizado varios choques sobre el carril, trataba de interpretarlos de manera unificada (Apéndice C).

La intención de $\mathrm{CE}$ era la de encontrar una causa única responsable de la continuidad del movimiento, de la detención o del rebote de la bolita incidente: la velocidad. CE, probablemente, había asociado los diferentes modos de lanzar el proyectil con las diferentes velocidades y con las diferentes «fuerzas de choque» $y$, en consecuencia, con los diferentes comportamientos; además pensaba que la diferencia de las masas incidentes podxía considerarse equivalente a una diferencia en las respectivas velocidades. La estudiante no resolvió el problema porque se cambió rápidamente de tema; sin embargo, ella misma retomó el conflicto en la segunda entrevista.

-El conflicto en paralelo es un proceso en el cual el estudiante desarrolla un discurso regulado por su conflicto efectivo y el interlocutor trata de profundizar sobre otras divergencias objetivas. EI caso de FE durante la segunda entrevista es, en este sentido, muy interesante (Apéndice C).

Para el estudiante, el conflicto de base, que domina su forma de responder a las preguntas de la entrevistadora, es que la bolita de acero rebota como la de goma, pero no se deforma durante el choque. Para la entrevistadora, en cambio, la preocupación fundamental es la clatificación de la fenomenología de los choques, evidentemente mal tratada ya sea en las previsiones como en las explicaciones del estudiante.

-El conflicto en serie consiste en un desarrollo conceptual caracterizado por la sucesión de conflictos limitados en los cuales la resolución de uno provoca el comienzo de otro. Un ejemplo representativo es lo sucedido con NA durante la primera entrevista (Apéndice C).

El estudiante no lograba conciliar la igualdad de acción y reacción, aprendida en su momento en la escuela, con la percepción de la velocidad alcanzada por el proyectil y el blanco después del choque en $c-5$. Su comportamiento posterior es un modelo de intento de salvar teoría y experimento, mediante la introducción de sucesivas hipótesis, las que fueron puestas sistemáticamente en discusión por la entrevistadora y luego descartadas por el estudiante, hasta su duda final sobre la validez efecti- va del principio de acción y reacción. El conflicto terminó con la intervención de la entrevistadora, que puso el acento en el concepto de aceleración y en la proporcio. nalidad inversa entre la variación de las velocidades y las respectivas masas.

-El conflicto en espiral, finalmente, se refiere a una situación conflictiva afrontada parcialmente, abandonada y sucesivamente reconsiderada en condiciones más apropiadas. Un ejemplo típico es el caso de THA, al comienzo de la primera entrevista (Apéndice C). La estudiante, frente a un resultado inesperado, elaboró y desarrolló una hipótesis de transmisión de la energía que la condujo a reconocer su incapacidad para explicar todos los fenómenos observados. La desviación del discurso sobre otro fenómeno permitió recuperar la idea de reacción, que rápidamente utilizó para completar la explicación anterior; fínalmente, una segunda desviación de la discusión le permitió efectuar un ulterior paso hacia adelante en la solución del primer conflicto.

\section{Las soluciones de los conflictos}

Una última característica didácticamente importante de los conflictos es el modo de terminarlos. Dos son los aspectos que han llamado nuestra atención: las modificaciones introducidas por el conflicto y su grado o nivel de conclusión.

\section{A. Las modificaciones}

Se definieron tres categorías: modificaciones no relevantes, parciales y profundas.

-Modificaciones no relevantes: se verifican cuando no aparecen las dificultades de la teoría del estudiante y ni siquiera aparecen nuevas relaciones, como sucedía cuando el estudiante iñventaba una explicación adhoc sin ninguna consecuencia futura. Por ejemplo, NA explicó el resultado de $\mathrm{p}-2$ con bolitas de goma y de acero, una vez con la deformación de la goma y la otra con la propiedad del acero de transmitir el choque sin deformarse. Hemos clasificado también en esta categoría los casos en que el conflicto terminaba con el reconocimiento de parte del estudiante que el problema no tenía solución, como sucedió con DA cuando debió reconocer que en el péndulo no podía existir un "choque débil», pero ignorando completamente la causa.

-Modificaciones parciales: se verificaban cuando las soluciones ponían en evidencia las dificultades de Ia teoría «vieja» o bien algunos aspectos de la «nueva» teoría.

En ei primer caso, frente a un resultado contradictorio, se abandonaba una relación anteriormente considerada general. Esto sucedió con CE, cuando frente al resultado de c-1 debió admitir que la transmisión parcial de energía del proyectil al blanco no era la única alternativa. En esta categoría hemos incluido también los casos en los cuales una idea espontánea muy arraigada era puesta en eviden- 
cia gracias a la intervención de la entrevistadora, como sucedió en la primera entrevista con NA, en la cual el resultado final fue la observación, aceptada por el estudiante, de la identificación implícita anterior entre velocidad y aceleración.

La revelación de aspectos de la nueva teoría sin el consecuente abandono de la vieja podía corresponder tanto a una relación nueva entre las variables en juego (de igual manera que cuando DA logró asociar el lanzamiento fuerte y débil de las bolitas de acero con las fuerzas de acción y reacción durante el choque) como a la nueva manera de analizar los experimentos, destacando por ejemplo el momento del choque y las consecuentes modificaciones, en lugar de referirse simplemente a las velocidades de lanzamiento.

- Modificaciones profundas: se observaron en los casos en que algún aspecto de la vieja teoría fue sustituido por el correspondiente aspecto de la nueva teoría. Hemos considerado en esta categoría tanto el caso de la aceptación plena de una nueva idea eliminando las resistencias como el abandono de una idea espontánea profundamente arraigada.

Un ejemplo significativo fue la aceptación, por parte de NA, de la deformación del acero sugerida por la entrevistadora, cuando consiguió percibir las similitudes entre los efectos con las bolitas de goma y las de acero.

Otro ejemplo es el del abandono por parte del mismo NA de la identificación de la conservación de la energía con la transmisión total del movimiento, introduciendo Ia consideración simultánea de los dos cuerpos en interacción. También podemos considerar, como transformación profunda, la sustitución, por parte de FE, de la fenomenología inicial compuesta de sólo dos categorías (continuación o inversión del movimiento) por una nueva fenomemología en la que se tenía en cuenta también la detención de la bolita incidente.

\section{B. El nivel de conclusión}

Hemos definido dos categorías: soluciones en suspenso y soluciones conclusivas. En el primer caso el conflicto acaba dejando en suspenso la posibifidad de un desarrollo ulterior hacia la visión disciplinar; en el segundo caso, el conflicto termina con un resultado que constituye, al menos para el estudiante, una síntesis definitiva.

-Soluciones en suspenso. Una forma en que se puede acabar el conflicto es mediante una pregunta dejada en suspenso, es decir, mediante el reconocimiento que el conflicto no tiene, por el momento, solución. Un ejemplo es la duda final de NA sobre la validez de la acción y la reacción en los experimentos en los que el proyectil rebota. Otro es la constatación DA, por parte de la imposibilidad de un choque débil en el péndulo y su reconocimiento de ignorar la causa.

Una segunda manera de acabar un conflicto es abandonando, al menos en parte, una relación antes considerada general sin especificar las condiciones de validez, o inventando una explicación ad hoc que no se deriva de las consideraciones anteriores ni tampoco indica consecuencias ulteriores; este tipo de solución no constituye una síntesis gemuina de un nuevo conocimiento del estudiante ni lo prepara para pasos ulteriores más relacionados con el plano disciplinar. El caso de $\mathrm{CE}$ es significativo: la estudiante estaba convencida de que no podía haber una transferencia total de la energía, pero el resultado de c-1 la sorprendió y debió admitir que, de hecho, la transferencia parcial no era la única posibilidad. Sin embargo su elaboración se detuvo en este reconocimiento, sin establecer ulteriores condiciones de aplicación; en algunas situaciones posteriores aplicó nuevamente la transferencia parcial y en otras no: por ejemplo, explicó que en p-2 las bolitas no habían alcanzado la altura inicial debido a la pérdida en el choque, mientras que el resultado de p-3 lo interpretó como un intercambio total de las velocidades entre las bolitas que colisionaban.

-Soluciones conclusivas. Consideramos conclusivos aquellos casos en los que el problema que había generado el conflicto fue resuelto por el estudiante mostrando la existencia de una relación nueva entre las variables en juego. Los conflictos de THA y de NA en la primera entrevista son un ejemplo de avance hacia el conocimiento disciplinar, después de un conflicto originado por un resultado experimental imprevisible. También la aceptación por parte de THA de la sugerencia de analizat la fase de interacción es un buen ejemplo de solución conclusiva. Durante el análisis de los choques sobre el carril, la estudiante inicialmente buscaba la explicación del comportamiento del proyectil después del choque en la velocidad inicial del mismo y en la fuerza de lanzamiento, mientras la entrevistadora orientaba sus preguntas hacia el momento del choque y las fuerzas de interacción. La insistencia de la entrevistadora consiguió convencer a la estudiante de concentrar el análisis en el momento de la interacción, arribando luego a resultados interesantes como el descubrimiento de la función de la reacción en la detención del proyectil.

Consideramos soluciones conclusivas también aquéllas que terminaban reforzando una idea espontánea del estudiante, como en el caso de que usó la idea de DA, reacción del blanco sobre el proyectil para justificar definitivamente la idea espontánea de una pérdida inevitable durante el choque.

\section{LA CONTRIBUCIÓN DIDÁCTICA DE LA ENTREVISTADORA}

El análisis de la gestión de la entrevista por parte de la entrevistadora constituye un complemento significativo para clarificar la compleja dinámica del desarrollo conceptual de los estudiantes entrevistados. Con este objetivo analizaremos con mayor detalle su contribución didáctica aportando informaciones suplementarias sobre las estrategias implícitas adoptadas por ella (Esquema 2). 
Uno de los resultados más importantes de este trabajo se refiere a la progresiva clarificación de la función de la entrevistadora como mediadora en el desarrollo de los conflictos cognitivos mediante tres tipos de actuaciones: transformar divergencias objetivas en conflictos efectivos, intervenir en los conflictos para aprovechar sus posibilidades y conducirlos en modo eficiente, y finalmente retomax las soluciones en suspenso transformándolas en conclusivas mediante la introducción de modificaciones significativas.

\section{Transformar las divergencias objetivas en conflictos efectivos}

La importancia didáctica de los conflictos objetivos y de la caracterización de la toma de conciencia de los estudiantes se pone en evidencia claramente cuando se analiza su desarrollo cognitivo: varios conflictos objetivos se transformaron con mucha facilidad en conflictos efectivos, pero en la medida en que la fuente del conflicto era más abstracta o sofisticada, la toma de conciencia por parte del estudiante era más complicada. En estos casos aumentaba la probabilidad de que el conflicto quedase sólo a nivel objetivo, ya sea porque el estudiante no tomaba en consideración la divergencia, ya sea porque se limitaba a percibir sólo sus aspectos parciales.

El problema de transformar un conflicto objetivo en uno efectivo, en general, requería de dos operaciones a veces simultáneas: indentificar las divergencias cognitivas, seleccionando aquéllas capaces de ser transformadas en conflicto; y organizar una secuencia didáctica adecuada para recuperar las residuales.

- La identificación de las divergencias objetivas era una tarea relativamente sencilla para la entrevistadora cuando el origen de éstas era externo, sobre todo si se trataba de diferencias entre previsión y resultado experimental. En estos casos la entrevistadora llamaba la atención del estudiante sobre los detalles de la previsión, o le pedía que repitiera el experimento, o le sugería que prestara mucha atención al resultado. En el caso de múltiples puntos de divergencia, éstos eran afrontados uno des. pués de otro o bien eran reconsiderados en situaciones más propicias cuando el estudiante manifestaba una mayor aproximación al conflicto. Por ejemplo, durante la entrevista con PA, no sólo sus previsiones sino también sus explicaciones presentaban incongruencias; la táctica de la entrevistadora fue presentarle un nuevo

Esquema 2. Contribuciones didăcticas de la entrevistadora.

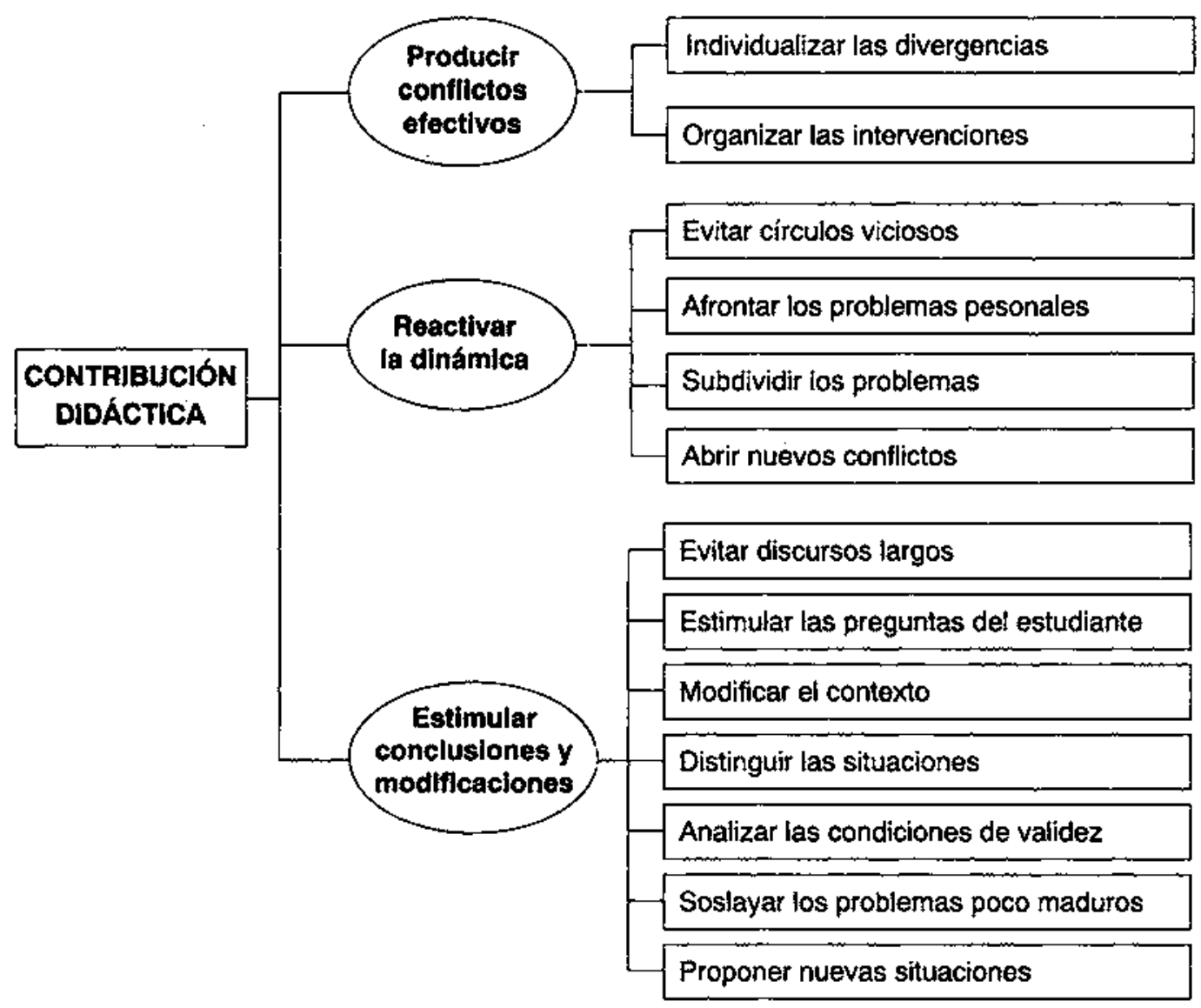


experimento cada vez que el estudiante reconocía la diferencia entre previsión y resultado experimental, sin detenerse en la discusión de las explicaciones dadas (en la esperanza de que al final pudiera realizarse una discysión más completa del modelo utilizado por el estudiante). Lamentablemente esta táctica se vio frustrada por la decisión repentina del estudiante de abandonar totalmente su modelo explicativo y adoptar la actitud más pragmática de apoyarse solamente en los indicios proporcionados por la entrevistadora.

La tarea didáctica resultaba mucho más complicada cuando estaban en juego divergencias internas. En algunas situaciones se observa claramente que la entrevistadora no logró percibir la amplitud de las perspectivas del estudiante, perdiendo la ocasión de una intervención significativa, como en el caso del conflicto de CE entre sus explicaciones sobre el movimiento del proyectil en los experimentos con el carril y sus exigencias de generalización manifestadas mediante una pregunta aparentemente poco significativa. En otras situaciones, en cambio, el conflicto objetivo fue rápidamente identificado, como en el caso de la divergencia entre la idea de acción y reacción aprendida por NA en la escuela y su explicación de la diferencia entre c-1 y c-2 atribuida a una mayor o menor fuerza de Ianzamiento: la intervención de la entrevistadora fue, primero, una pregunta de confrontación entre c-1 y p-1, rápidamente resuelta por el estudiante al darse cuenta de la analogía en la transferencia en los dos choques, y luego la sugetencia de un nuevo experimento (c.5) para poner en evidencia el problema de la reacción.

- La organización de las intervenciones de la entrevistadora, en general, exigía que las divergencias fueran retomadas todas las veces que la situación lo permitiera ( $\sin$ forzar el desarrollo del pensamiento del estudiante); esto requería mucha atención a las ideas del estudiante por parte de la entrevistadora, quien debía además interpretar rápidamente los indicios por él proporcionados y dar preferencia a las divergencias más factibles de transformarse en efectivas.

La situación de conflicto incipiente constituye un caso especial en el cual la divergencia de perspectivas entre profesor y estudiante no Ilega a ser visualizada por el estudiante, que gira en torno al problema sin afrontarlo. Se encontró una posibilidad de desarrollo eficiente en Ia construcción lenta đe una sólida base común de điálogo, a partir de la cual fuera posible establecer eventos y relaciones bien definidas, antes de que surgiera con fuerza una confrontación entre posiciones diferentes o resultaran inteligibles preguntas precisas con respecto a eventos problemáticos. El caso de $\mathrm{FE}$, en la primera entrevista, cuando estaba en juego una descripción adecuada de la fenomenología de los choques en el péndulo y en el carril, nos parece que muestra la importancia de saber esperar por parte de la entrevistadora a pesar de los errores que cometió insistiendo, algunas veces inútilmente, con preguntas que tenían poco significado para el estudiante. El conflicto resultó significativo para el estudiante sólo en la segunda entrevista, al tomar la forma de una pregunta que le creaba problemas (la causa del retorno del proyectil de acero), pero que le permitió englobar e integrar varias informaciones anteriormente desechadas.

\section{Intervenir en la dinámica de los conflictos}

Para poder intervenir constructivamente en los conflictos, la entrevistadora debía prestar atención a la đinámica de los mismos. El problema más importante por resolver era entrar en resonancia con el conflícto del estudiante.

-Evitar la impotencia cognitiva. Algunas situaciones de conflicto local eran aprovechadas por la entrevistadora como ocasiones en las cuales el estudiante pudiera usar todas sus habilidades de resolución, formulando hipótesis y discutiéndolas; lo importante era no permitir que el estudiante terminase en un círculo vicioso. El caso de $\mathrm{CE}$, citado anteriormente como un ejemplo de conflicto local, muestra un comportamiento didáctico poco feliz, ya sea porque la entrevistadora no supo retomar enseguida, durante la primera entrevista, la exigencia de generalización y unificación de la estudiante (probablemente pensando que se trałaba de una exigencia débil), ya sea porque en la segunda entrevista, cuando la estudiante retomó por sí sola el problema, aun así, su exigencia intelectual fue ignorada en favor de otro conflicto. Un ejemplo, en cambio, de intervención didáctica eficaz, en la cual un conflicto local aparentemente cerrado y resuelto fue reabierto naturalmente conduciendo a una nueva conclusión, es el de CE, durante la primera entrevista, cuando le surgió el conflicto entre la idea de un choque p 1 ideal y la necesidad, aprendida en clase, de una pérdida de energía transformada en calor durante los choques. La estudiante desarrolló el conflicto por sí sola y conciuyó que en el caso de p-1, el proyectíl no podía transferir toda su energía al blanco. La entrevistadora desvió el análisis sobre la diferencia entre choques elásticos y no elásticos, concluyendo con la demostración de un choque c-1; el resultado sorprendió a $\mathrm{CE}$ obligándola a admitir, por primera vez, que toda la energía podía pasar del proyectil al blanco.

-Afrontar los conflictos del estudiante. La dificultad de los conflictos en paralelo consistía principalmente en la falta de percepción por parte de la entrevistadora del conflicto en el que estaba inmerso el estudiante. Cuando esto aparecía en forma explícita la entrevistadora podía ayudar al estudiante en la solución de su problema, retomando luego al menos una parte de los conflictos antes dejados de lado. En el ejemplo de $\mathrm{FE}$ citado anteriormente (Apéndice C), la entrevistadora se encontró varias veces frente a divergencias objetivas orientando el diálogo en la dirección de hacerlas explícitas; solamente al finai comprendió el problema efectivo que el estudiante quería resolver y pudo ayudarlo.

- Reformular el problema en etapas. Cuando el conflicto comprometía directamente ideas espontáneas que resultaban fundamentales en la concepción de los choques, la reestructuración profunda de las ideas del estudiante 
exigia, para poder realizarse, que el problema fuera suficientemente maduro. Es el caso de los conflictos en espiral, como, por ejemplo, la confrontación de THA entre su modelo de acción y reacción y el de transferencia de energía. En estas situaciones la entrevistadora trataba de elaborar una estrategia en etapas sucesivas, caracterizadas cada una de ellas por un menor grado de reestructuración, con el fín de abrir la posibilidad de soluciones progresivamente más estructuradas.

-Abrir nuevos conflictos. Cuando la entrevistadora percibía que $\mathbf{l}$ l estudiante estaba lo sufucientemente seguro para aceptar una intervención más decisiva, el diálogo se hacía más cerłado y las preguntas se sucedían abriendo continuamente nuevos conflictos, como demuestra el ejemplo de los conflictos en serie descrito en el apéndice.

\section{Conducir hacia modificaciones profundas y soluciones conclusivas}

Las estrategias didácticas utilizadas por la entrevistadora se evidencian también en el modo de afrontar la resolución de los conflictos propuesta por los estudiantes. El esfuerzo implícito de la entrevistadora estaba dirigido a encontrar una solución conclusiva que implicase la sustitución de una idea espontánea.

-Evitar el exceso de información. Este objetivo ha sido bastante difícil de obtener: en algunas ocasiones la entrevistadora parecía querer agregar información a toda costa, sin tener en cuenta en qué medida era significativa para el estudiante.

-Estimular las preguntas del estudiante. Algunas veces, en cambio, intervenía para valorar en qué medida el problema era significativo para el estudiante, por ejemplo, ofreciéndole la posibilidad de hacer preguntas. PA y FE aprovecharon esta oportunidad al comienzo de la segunda entrevista para aclarar dudas que tenían de la sesión anterior

- Soslayar los problemas poco maduros. Varias veces la entrevistadora se encontró frente a una solución en suspenso y decidió soslayarla provisionalmente en espera de desarrollos futuros más promisorios. En particular, cuando se encontraba frente a ideas recurrentes que el estudiante no conseguía superar, utilizaba una táctica interesante que era la de ayudarlo a individualizar las posibles situaciones en las cuales sus ideas estuvieran de acuerdo con la descripción científica acreditada, evitando así que las dudas continuaran siendo una fuente de conflicto reprimido y retomando lyego los puntos dejados en suspenso.

-Distinguir las situaciones problemáticas. Es importante destacar que frente a soluciones en suspenso era necesario distinguir la naturaleza de la duda que mantenía el estudiante. Los dos casos de DA y NA citados con anterioridad como ejemplos de soluciones en suspenso representan situaciones cognitivamente diferentes a pesar de la similitud aparente del reconocimiento de la incapacidad de los estudiantes. De hecho, el problema de DA (¿por qué no existen choques débiles en el péndulo?) es específico, basado en la noción personal de choque "débít» que no tenía en cuenta la rotación del proyectil antes del choque. En cambio, el problema de NA es más general, basado en la representación espontánea de la segunda ley de Newton. Consecuentemente, en el caso de NA, la profundización de la relación $\mathrm{f}=\mathrm{m}$.a provocó una respuesta inmediata del estudiante, mientras que en el caso de DA la entrevistadora no logró retomar el conflicto durante la primera entrevista y, prácticamente tuvo de comenzar todo de nuevo en la segunda.

-Insistir en las condiciones de validez. La entrevistadora tuvo dificultad en aplicar la estrategia de presionar al estudiante para que definiera las condiciones de validez de sus afirmaciones. Por ejemplo, cuando finalmente CE admitio que la energía podía ser transferida totalmente del proyectil al blanco, la entrevistadora no profundizó en el tema a fin de llegar a definir en qué circunstancias se verificaba esta regla. En consecuencia, como ya hemos visto anteriormente, $\mathrm{CE}$ continuó utilizando la regla antigua (imposibilidad de transferencia total ) en p-2 y la nueva (posibilidad de transferencia total) en $p-3$, sin un criterio definido de selección. En algunos casos se obtenía un mayor control de la situación aumentando el análisis experimental con el fin de obtener una mayor familiaridad del esudiante con el fenómeno como paso importante para poner en evidencia las diferentes condiciones de contorno y reorganizar los casos en los que funcionaban la vieja y la nueva relación.

-Proponer nuevas preguntas y nuevas situaciones. Cuando la entrevistadora se daba cuenta de que el estudiante manifestaba dudas que parecían haber sido resueltas precedentemente, con frecuencia utilizaba la estrategia de proponer un análisis global de todos los casos analizados, con la intención de ofrecer un cuadro en el cual se pudieran manifestar y posiblemente resolver las dudas, como sucedió con PA y DA en la segunda entrevista.

También en los casos de soluciones conclusivas alcanzadas mediante la introducción de una nueva relación entre las variables en juego, a veces resultaba muy útil hacer una profundización mediante nuevas preguntas y nuevos experimentos para controlar la efectiva asimilación de la nueva idea.

Esta exigencia era mayor cuando la conclusión del conflicto se había logrado mediante la aceptación de una sugerencia de la entrevistadora. Un caso ejemplar es el de la aceptación de la idea de deformación del acero por parte de THA: la entrevistadora explotó ampliamente este nuevo conocimiento llegando a obtener nuevos progresos.

- Modificar el contexto de los problemas. Cuando el estudiante llegaba a una conclusión que reforzaba sus ideas espontáneas, la entrevistadora trataba de poner en duda tal resultado, pero esta tarea no podía ser afrontada directamente; trataba entonces de modificar el contexto y conducir la discusión a nuevas conclusiones que eran posteriormente confrontadas con las anteriores. 


\section{ALGUNAS CONCLUSIONES Y CONSIDE- RACIONES DIDÁCTICAS}

Nuestro análisis de los conflictos y de las intervenciones de la entrevistadora nos parece totalmente compatible con los resultados recientes referentes a las reacciones de los estudiantes ante datos incompatibles con sus ideas (Chinn y Brewer, 1993); sin embargo las estrategias didácticas sugeridas por estos autores parecen ser de largo alcance y encaminadas al cambio conceptual, mientras que en nuestro caso se refieren simplemente al desarrolio de los conflictos. Creemos haber conseguido documentar varios casos de éxito y de fracaso, interpre. tándolos desde una perspectiva constructivista de enseñanza-aprendizaje.

a) Nuestra conclusión más general se refiere a la compleja dinámica de los conocimientos de los estudiantes durante las entrevistas. El bagaje cognitivo personal (ideas espontáneas, cultura científica y ecología conceptual), las evidencias experimentales y las intervenciones de la entrevistadora representan tres fuentes diferentes, aunque interdependientes, de conflictos que deberían ser armonizadas para que el estudiante se desarrolle en la dirección del conocimiento científico.

Cuando no se tenía en cuenta el bagaje cognitivo de los estudiantes, el diálogo entre la entrevistadora y el estudiante perdía progresivamente significado y la posibilidad de influir en el conflicto se desvanecía rápidamente. Por otra parte cuando se renunciaba a la posibilidad de proporcionar informaciones científicas precisas se corría el riesgo de desarollos que se alejaban del aprendizaje de conocimientos científicos acreditados, y luego resultaba difícil retomar los conflictos dejados en sus. penso para transformarlos en soluciones conclusivas. Las informaciones implícitas o explícitas dadas por la entrevistadora, a su vez, resultaron muy útiles para abrir el camino bacia ideas a primera vista extrañas o poco inteligibles para los estudiantes, sobre todo durante el proceso de identificación de los puntos esenciales; los conflictos generados por las sugerencias de la entrevistadora resultaban con frecuencia efectivos debido al peso de su autoridad. Finalmente, los experimentos simples demostraron ser una fuente muy importante de conocimientos y un potente punto de partida para desencadenar conflictos efectivos; la introducción de una actividad de exploración experimental garantizaba casi siempre la existencia de un referente fenomenológico objetivo y facilitaba la confrontación entre proposiciones diferentes (teóricas y experimentales) (Villani y Urquiza, 1993).

b) Una característica que nos ha llamado la atención analizando las entrevistas es la variabilidad de los resultados efectivos producidos a partir de actividades similares, los cuales, en algunos casos, tenían un éxito evidente y, en otros, no contribuían demasiado al desa rrollo cognitivo del estudiante, aunque el objetivo y la perspectiva de la entrevistadora fueran idénticos. Consideramos que los datos recogidos sugieren una atención especial a la resonancia didáctica entre las iniciativas de la entrevistadora y la respuesta del estudiante. Cuanto mayor era la disponibilidad del estudiante, como por ejemplo en el caso de THA y NA, que poseían una mayor cultura científica, cualquier sugerencia de la entrevistadora era considerada inmediatamente como objeto de análisis, aunque no perteneciera a sus campos de interés específicos o no interrumpiera un conflicto; sin embargo, las soluciones obtenidas eran, en algunos casos, de una estabilidad limitada.

En cambio, cuando la disponibilidad del estudiante era menor, como sucedía con DA y con $\mathrm{CE}$, que preferían desarrollar sus propias ideas, el problema era insertarse en el conflicto del estudiante orientando su atención hacia aspectos que debían ser elaborados o modificados. Por esto, creemos poder concluir que la habilidad de la entrevistadora se manifestaba en dos etapas: inicialmen. te debía percibir en qué medida podía modificarse el punto de vista del estudiante, a fin de elegir entre un desarrollo exhaustivo de las ideas del estudiante o una insistencia en la propia perspectiva hasta lograr la aceptación por parte del estudiante. Luego debia concluir el trabajo desarrollado orientando al estudiante hacia el logro del conocimiento científico o garantizando la profundización del conocimiento adquirido.

c) Creemos poder concluir también que la resonancia cognitiva entre los elementos conflictivos en discusión y el dominio conceptual del estudiante constituye un filtro que determina implícitamente las posibilidades intrínsecas de desarrollo cognitivo. Cuando los elementos en discusión (por ejemplo la importancia de lo que sucede en el momento en que se produce el choque) se alejaban mucho de las perspectivas de los estudiantes (por ejemplo, FE que consideraba el choque entre péndulos como un proceso global) era muy improbable lograr un desarrollo cognitivo, independientemente del esfuerzo de la entrevistadora. En este caso, su habilidad para introducir conflictos efectivos o bien, para introdu. cirse en su dinámica, dependía de su capacidad de modificar los elementos en juego, de manera que resultaran más significativos para el estudiante y desencadenaran un proceso por etapas parciales sucesivas. En el ejemplo citado, la introducción de la semejanza entre los choques realizados con péndulos y aquéllos realizados en el carril, representó un tipo de mediación eficiente, que posibilitó la aparición de las variables significativas de los choques.

d) Finalmente, la función de la entrevistadora como fuente de información merece una consideración especial. Nuestro análisis sugiere que el problema es complejo. Ciertamente, las intervenciones breves, que favorecían el desarrollo de la entrevista, focalizando mejor los problemas o introduciendo hipótesis claramente ligadas al contex to y fácilmente comprensibles, constituyeron la levadura para el desarrollo intelectual de los estudiantes. En cambio, las intervenciones que proporcionaban in. formaciones estructuradas tuvieron efectos ambiguos. Toda vez que tales intervenciones estaban precedidas 0 seguidas de un esfuerzo de problematizar las nociones en juego, los estudiantes lograban aplicarlas con mayor facilidad y competencia; cuando en cambio, las informaciones superaban el dominio de la problematización, los 
estudiantes parecían no ser capaces de valorar su plausibilidad y utilidad. Análogamente, la eficacia didáctica de la repetición de las observaciones experimentales parece estar ligada a la presencia de un problema a resolver (Gil et al., 1988), que permitió a la entrevistadora llamar la atención sobre las semejanzas y diferencias, ayudando a los estudiantes a pensar por analogía, a asociar el choque con las características del sistema en interacción y a dejar de lado progresivamente los detalles científicamente poco significativos.

En la literatura existe una tendencia a revalorizat la función del docente como fuente de información; nues. tras conclusiones están de acuerdo con las de otras investigaciones anteriores (Dreyfuss et al., 1990; Sere, 1992; Jung, 1992) en cuanto a la ambigüedad de la eficacia de proporcionar explicaciones demasiado estructuradas. Nos parece importante además destacar la fertilidad de las informaciones implícitas dadas por la entrevistadora al estudiante en la realización de los experimentos y de las discusiones, con respecto sobre todo al valor cognitivo de la generalización, a la coherencia de las explicaciones, a la compatibilidad entre teoría y experimento. Por otra parte, las informaciones sobre la identificación y el control de las variables significativas, sobre la diferencia entre situación ideal y real, sobre las condiciones de contorno significativas, eran, de hecho, transmitidas implícitamente al estudiante toda vez que la entrevistadora fijaba deliberadamente sobre ellas el objetivo del análisis. Algunas modificacio-

\section{REFERENCIAS BIBLIOGRÁFICAS}

BAIRD, J.R. Y MITCHELL, I. J. (eos.) (1986). Improving the Quality of Teaching and Learning: An Australian Case Study. The PEEL Project. Melbourne: Monash University.

BROWN, D. y CLEMENT, J. (1992). Classroom teaching experiments in mechanics, en Duit, R., Goldberg, F.,Niedtierer, H. (eds.), Research in Physics Learning: Theoretical Issues and Empirical Studies. IPN. Kiel (D), pp. 380-397.

CHINN,A.C. y BREWER, W.F. (1993). The Role of Anomalous Data in Knowledge Acquisition: A Theoretical Framework and Implications for Science Instruction . Review of Educational Research, 63(1), pp. 1-49.

DREYFUS, A., JUNGWIRTH, E. y ELIOVITCH, R. (1990). Applying the 'Cognitive Conflict' strategy for conceptual change: Some implications, difficulties and problems. Science Education, $74(5)$, pp. 555.569 . nes de comportamiento de los estudiantes de la primera a la segunda entrevista parecen atribuibles al efecto de esas informaciones implícitas.

Sin embargo, para concluir, nos parece que hay aún un largo camino para recorrer, sobre todo en lo que se refiere a la interacción de los estudiantes entre ellos y con el profesor, antes de alcanzar la meta sugerida por Dykstra (1992) y por Niedderer y Schester (1992) de hacer plenamente inteligible el proceso de cambio conceptual que se produce en la clase.

\section{NOTAS}

- Este trabajo es una reelaboración de la comunicación presentada en eI IV Congreso Internacional sobre Investigación en la Didáctica de las Ciencias. Barcelona (España). Setiembre, 1993. Ha sido traducido del italiano por Silvia Gamero.

1 Con financiación parcial del CNPQ (Brasil).

${ }^{2}$ Con financiación parcial de la CAPES (Brasil).

${ }^{3}$ Una de éstas (la segunda con DA) no fue registrada adecuadamente y por ello fue muy poco utilizada.

${ }^{4}$ Nuestra clasificación presenta una semejanza evidente con las consideraciones de Chinn y Brewer (1993) y con las categorías piagetianas de conoucta alfa, beta y gama (Mariani, 1987; Rowell, 1989).
DRIVER, R. (1973). The representation of conceptual frameworks in young adolescent science students. Doctoral dissertation, University of Illinois, Urbana"Champaign.

DUSCHL, R.A. y GITOMER, D.H. (1991), Epistemological Perspective on Conceptral Change: Implications for Educational Practice.Journal of Research in Science Teaching, 28(9), pp. 839-858.

DYKSTRA, D. (1992). Studying conceptual change: Constructing new understandings, en Duit, R., Goldberg, F., Niedderer, H. (eds.), Research in Physics Learning: Theoretical Issues and Empirical Studies. IPN. Kiel (D), pp. 40-58.

GIL, D., MARTÍNEZ-TORREGROSA, J. y SENENT, F. (1988). EI fracaso en la resolucion de problemas: una investigacion orientada por nuevos supuestos. Ensenanza de las Ciencias, $6(2)$, pp. $131-146$. 
GRIMELLINI, T. N., PECORI, B.B., VILLANI, A., CASADIO, C., y PACCA, J.L.A. (1989). Teaching Strategies and Conceptual Change: The Case of Collisions in Mechanics. Paper presented at Annual Meeting of the American Educational Research Association, S. Francisco, CA.

GRIMELLINI, T.N.,PECORI, B.B.,PACCA, J.L.A. y VILLANI, A. (1993). Understanding conservation laws in mechanics: Students' conceptual change in learning about collisions. Science Education, 77(2), pp. 169-189.

HEWSON, P.W. (1985). Epistemological Commitments in the Learning of Science: Examples from Dynamics. European Journal of Science Education, 7, pp. 163-172.

HODSON, D. (1988). Experiments in science and science teaching. Educational Philosophy and Theory, 20(2), pp. $53-66$.

JUNG, W. (1992) Probing acceptance: A technique for investigating learning difficulties, en Duit, R., Goldberg, F., Niedderer, H. (eds.), Research in Physics Learning: Theoretical Issues and Empirical Studies. IPN. Kiel (D), pp. 278-295.

MALGRANGE, J. L., SALTIEL, E. y VIENNOT, L. (1973). Vecteurs, scalaire et grandeurs physiques. Bulletin de la Societé Française de Physique-Encart Pedagogiques, 1, pp. 3-13

MARIANI, M. C. (1987). Evolução das Concepçōes Espontáneas sobre Colisōes. Master Dissertation. Universidade de Sāo Paulo.

NACHTIGALL, D.K. (ed.) (1992). Proceeding of The International Conference on Physics Teachers' Education. Dortmund (Germany).

NERSESSIAN, N.J. (1989). Conceptual change in science and science education, Synthese, 80, pp. 163-183.

NIEDDERER, H. y SCHESTER, H. (1992). Toward an explicit description of cognitive systems for research in physics learning, en Duit, R., Goldberg, F., Niedderer, H. (eds.), Research in PhysicsLearning: TheoreticalIssues and Empirical Studies. IPN. Kiel (D), pp. 74-98.
NUSSBAUM, J. y NOVICK, S.(1982). Alternative Frameworks, Conceptual Conflicts and Accomodation: Toward a Principled Teaching Strategy. Instructional Science, 11, pp. 183-200.

POSNER, G.J., STRIKE, K.A.,HEWSON, P.W. y GERTZOG, W.A. (1982). Accommodation of a Scientific Conception: Toward a Theory of Conceptual Change. Science Education, 66(2), pp. 211-227.

ROWELL, J.A. (1989). Piagetian Epistemology, Equilibration and the Teaching of Science. Synthese, 80, pp. 141-162.

ROWELL, J. A. y DAWSON, C. J. (1985). Equilibration, Conflict and Instruction: A New Class-oriented Perspective European Journal of Science Education, 7, pp. 331-344.

SCOTT, P.H., ASOKO, H.M. y DRIVER, R.H. (1992). Teaching for conceptual change. A review of strategies, en Duit, R., Goldberg, F., Niedderer, H. (eds.). Research in Physics Learning: Theoretical Issues and Empirical Studies. IPN. Kiel (D), pp. 310-329.

SÉRÉ, M.G. (1992). Learning by giving and receiving explanations, en Dutit, R., Goldberg, F., Niedderer, H. (eds.), Research in Physics Learning: Theoreticallssues and Empirical Studies. IPN. Kiel (D), pp. 259-277.

STRIKE, K.A. y POSNER, G.J. (1992). A Revisionistic Theory of conceptual change, en Duschi y Hamilton (eds.): Philosophy os Science, Cognitive Science and Educational Theory and Practice. Albany, NY: SUNY Press.

VILLANI, A. (1992). Conceptual Change in Science and Science Education. Science Education, 76(2), pp. 223-237.

VILLANI, A. y ORQUIZA, L.C. (1993). Representaçōes mentais e Experimentos Qualitativos. Revista Brasileira de Ensino de Física, V. 15(1), pp. 74-89.

VILLANI, A. y PACCA, J.L.A. (1990). Spontaneous Reasoning of Graduate Students. International Journal of Science Education, 12(5), pp. 589-600 


\section{APÉNDICES}

\section{APÉNDICE A: LAS ACTIVIDADES DIDÁCTICAS DE LA ENTREVISTADORA}

Una de las principales actividades de la entrevistadora fue la de hacer preguntas. Además de las sistemáticas, relativas a la formulación de previsiones y a la descripción y explicación de los resultados de las experiencias, las preguntas más significativas se referían a las semejanzas y diferencias entre experimentos, a la compatibilidad entre las respuestas dadas por los estudiantes en diversos momentos de la entrevista, a la posibilidad de utilizar un determinado modelo en una nueva situación, a la posibilidad de producir determinados resultados y a las consecuencias de desechar algunas variables (no significativas).

La función de las preguntas formuladas por la entrevistadora era múltiple: poner al estudiante en una situación problemática compatible con sus posibilidades cognitivas, indicarle puntos especificos sobre los cuales reflexionat y sugerit un modo organizado de pensar. Simultáneamente e implícitamente se valorizaban determinados tipos de información y se mantenía el diălogo en una perspectiva científicamente acreditada.

Además la entrevistadora proporcionó informaciones breves a todos los estudiantes, sugiriendo hipótesis, haciendo comentarios respecto a las afirmaciones o recordando relaciones ya vistas oresuitados ya observados; en algunos casos también proporcionó explicaciones más amplias respondiendo a preguntas explícitas de los estudiantes o satisfaciendo necesidades implícitas. Las informaciones rápidas constituían una ayuda específica para el desarrollo conceptual de los estudiantes, mientras que con las explicaciones complejas se buscaba proporcionarles una visión global y sintética de los principios físicos más imporlantes.

La realización de experimentos constituyó el leit-motiv constante durante toda la entrevista: una vez montados los dispositivos experimentales, los experimentos eran sencillos de realizar y no requerían de una preparación específica. Aun cuando el estudiante había observado ya un resultado, la entrevistadora repetía el experimento varias veces durante el período en el cual se desarrollaba la discusión correspondiente, permitiendo así al estudiante reparar en detalles importantes de tener en cuenta o de desechar.

Muchas veces eta la entrevistadora quien solicitaba a los estudiantes que repitieran los experimentos. La actividad experimental tenía dos funciones: la primera era hacer que el estudiante se familiarizara con las características y los resultados del experimento. La segunda era la valoración conceptual y cognitiva de la actividad experimentah, debida sobre todo a la insistencia de la entrevistadora de obtener resultados fiables y de referirse a ellos.

La entrevistadora también pedía a los estudiantes que imaginaran nuevos experimentos, similares a los realizados, como por ejemplo choques con péndulos o sobre el carril con bolitas de plastilina. En algunos casos, además de las previsiones, la entrevistadora pedía que formularan conclusiones basándose únicamente en resultados hipotéticos, con la intención de valorizar aún más el proceso de razonamiento por analogia.

Otra actividad muy estimulante para los estudiantes fuse la de pedirles que formularan preguntas. Los resultados fueron interesantes porque las preguntas de los estudiantes pusieton en evidencia los puntos esenciales del confilicto todavía no resuelto. Las explicaciones posteriores de la entrevistadora parecen haber sido vitales para el estudiante.

\section{APÉNDICE B: LOS APARATOS Y LOS EXPERIMENTOS}

El dispositivo I estaba compuesto por un conjunto de péndulos de bolitas de acero de $50 \mathrm{~g}(\mathrm{M})$ o $100 \mathrm{~g}(\mathrm{G})$ sostenidos por una estructura de madera. Las bolitas podían ser quitadas y reemplazadas. Esto permitía reakizar varios ejemplos de choques (Fig.1):

Figura 1. Péndulo.

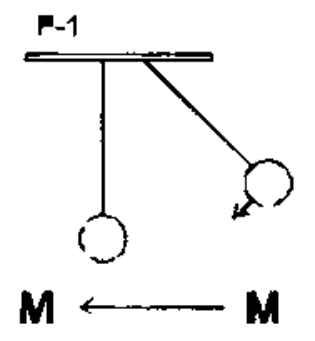

$+4$

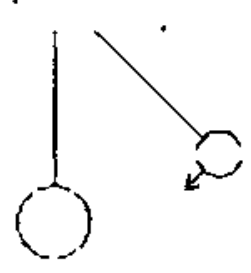

$\mathbf{G}<\quad \mathbf{M}$
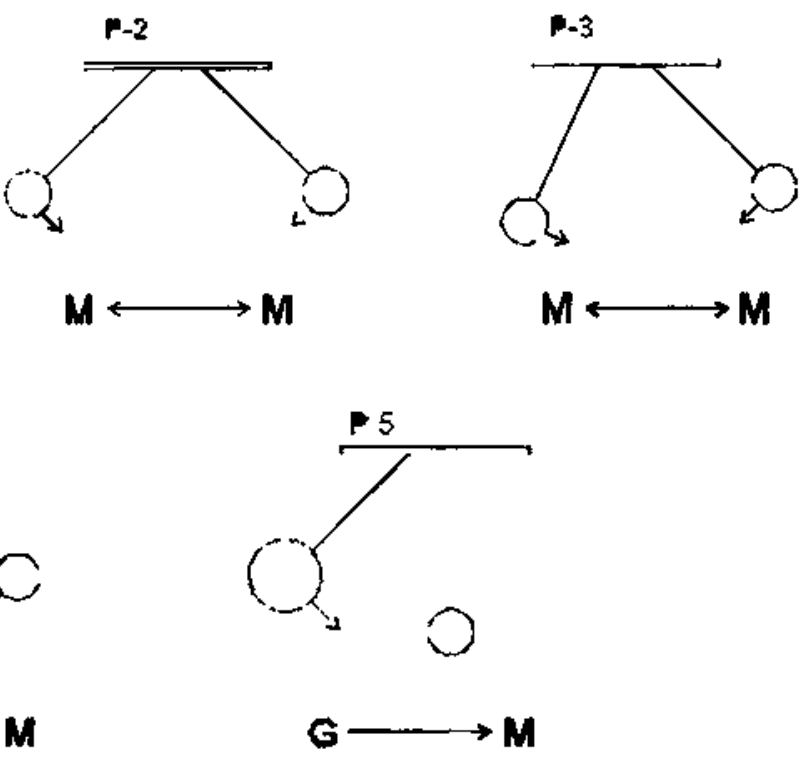
p-1 era tn choque $M \rightarrow M$, p-4 era $M->G$ y p-5 era $G$-> $M$; el blanco inicialmente estaba en reposo en posición vertical y el proyectil se dejaba caer desde una altura determinada.

En p-1 el proyectil se detenía y el blanco seguía prácticamente con la misma velocidad inicial del proyectil.

En p-4 el proyectil retornaba con poca velocidad y el b́lanco proseguía con una velocidad mayor (aunque inferior a la velocidad inicial del proyectil).
En p-5 el blanco continuaba con mayor velocidad y el proyectil también pero con una velocidad menor que la inicial.

Los casos p.2 y p-3 eran choques $G<->G$ o $M$ \& $M$.

En p-3 las bolitas se abandonaban a alturas diferentes y volvían invirtiendo sus alturas.

El dispositivo II estaba constituido por una carril plano de $1 \mathrm{~cm}$ de ancho y un conjunto de bolitas de acero de $25 \mathrm{~g}(\mathrm{P}), 50 \mathrm{~g}(\mathrm{M})$ y $100 \mathrm{~g}(\mathrm{G})$. Se podían realizar varios experimentos variando la masa de las bolitas y la manera de lanzarlas sobre el carril.

Figura 2. Carril - Deslizamiento.
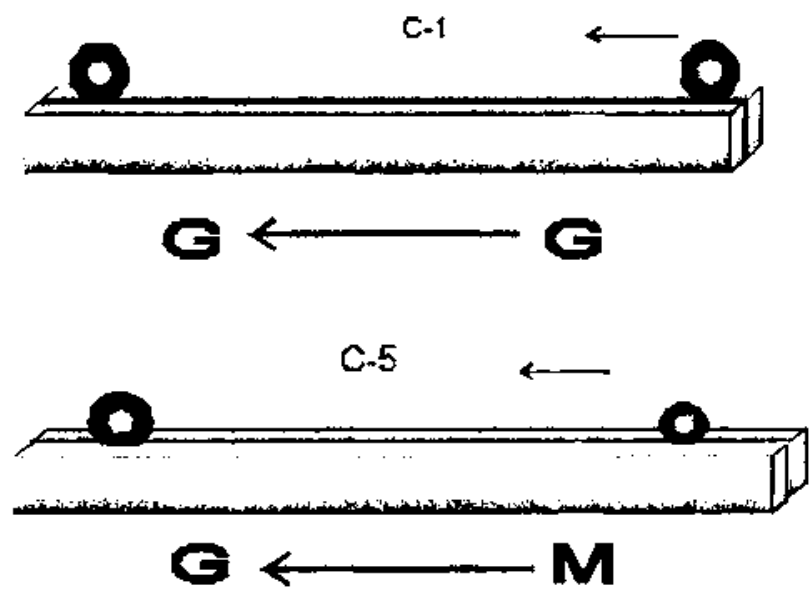
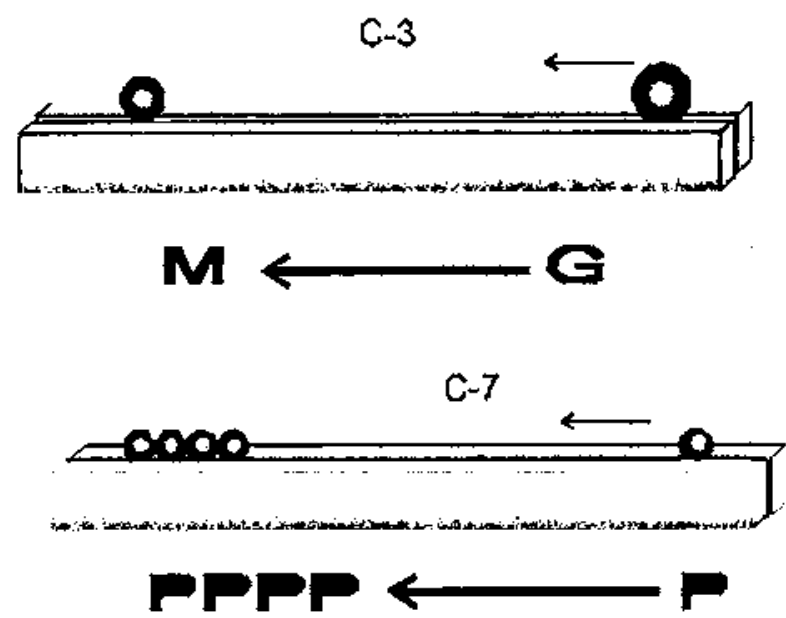

C-8

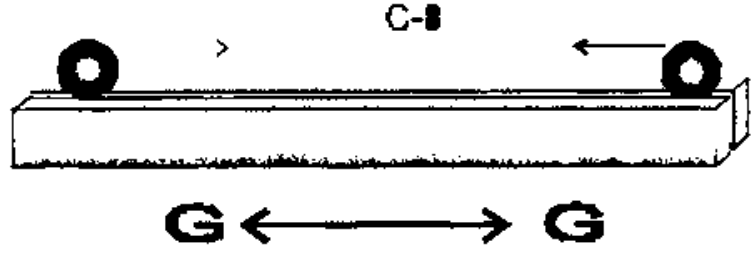

Cuando se lanzaban en modo seco y rápido, prácticamente se deslizaban hasta el momento del choque (Ftg. 2):

c-1 era el choque $\mathbf{G}>\mathbf{G}, \mathbf{c - 3}$ era $\mathrm{G} \rightarrow \mathrm{M}$ y $\mathbf{c}-5$ era $\mathrm{M} \rightarrow \mathrm{G}$; el blanco estaba inicialmente en reposo y el proyectil era lanzado a su encuentro deslizándose. El movimiento del proyectil y del blanco después del choque era prácticamente idéntico al de p-1, p-5 y p-4, respectivamente.

c-7 era un choque P $\rightarrow$ PPPP; el proyectil P se lanzaba deslizándose contra el blanco en reposo compuesto de cuatro o seis bolitas (P) colocadas una al lado de la otra. Algunas variaciones de c-7 eran: lanzar dos (PP) o tres (PPP) bolitas sobre el blanco. El resultado era que las bolitas que formaban el proyectil se detenían y un número igual de bolitas abandonaba el blanco con una velocidad similar a la del proyectil.

Cuando Ias bolitas se lanzaban con un poco de rotación continuaban rodando (Fig. 3). c-2 era un choque $G \rightarrow G$, c-4 era $G \rightarrow M$ y c-6 era $M->G$; eI blanco estaba inicialmente en reposo y el proyectil se lanzaba a su encuentro rodando.

En c-2 el proyectil continuaba rodando hacia adelante después de haberse detenido por un instante y el blanco continuaba con una velocidad un poco inferior a la inicial del proyectil.

En c-4 el proyectil y el blanco continuaban rodando hacia adelante después del choque, uno con velocidad inferior y el otro con una velocidad superior a la inicial del proyectil.

En c-6 el blanco continuaba con velocidad inferior a la inicial del proyectil; éste volvía hacia attás un poco y luego se detenía o retomaba el movimiento con un pequeña velocidad hacia adelante, según el modo en que había sido lanzado y el choque.

Los casos $c-8$ y c-9 eran choques $G \leftrightarrow->$; las bolitas se lanzaban con la misma velocidad una contra la otra con 
Figura 3. Carril- Rodamiento.
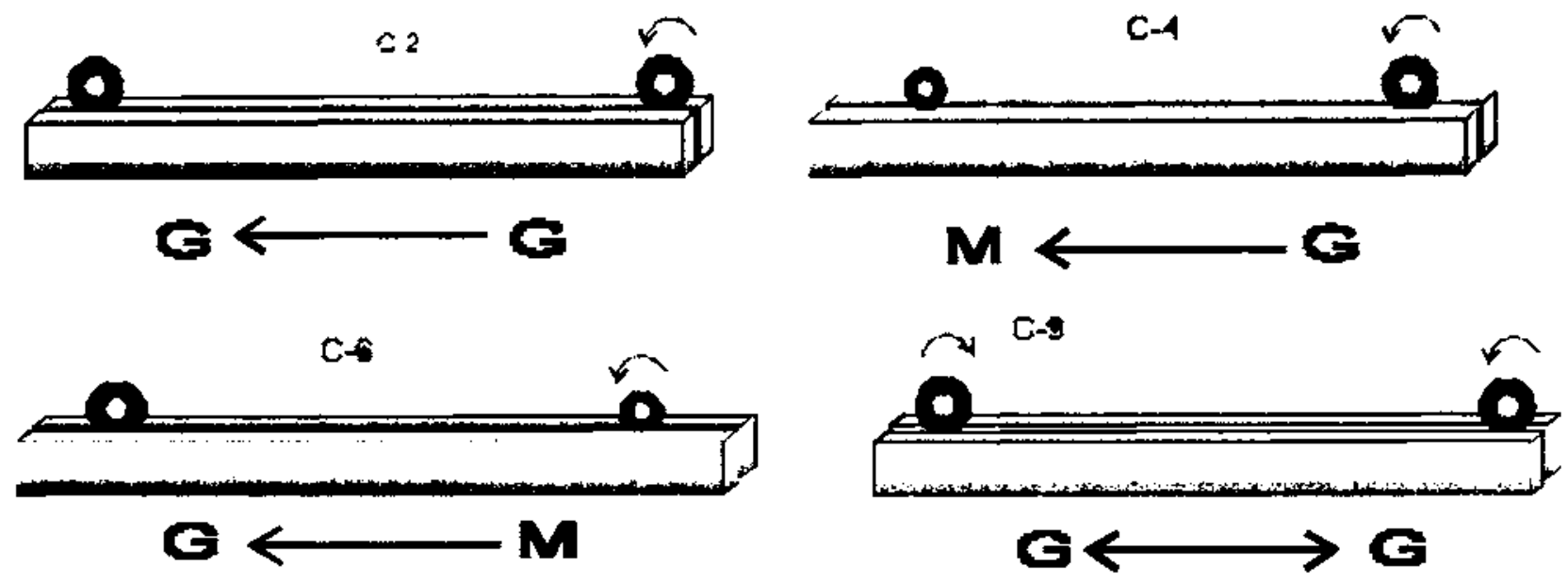

deslizamiento (c-8) y rodando (c-9); volvían hacia atrás con velocidades iguales pero inferiores a la velocidad de lanzamiento.

EI dispostitivo III estaba constituido por algunas bolitas de plastilina, goma dura, goma blanda y acero y por algunas planchas de plastilina, madera y esponja. Servía paris realizar o para imaginar choques elásticos y no elásticos.

El dispositivo IV estaba formado por dos carritos con una masa de $100 \mathrm{~g}$ cada uno con un muelle comprimido que podía soltarse, una guía con un sostén y algunos bloques de $100 \mathrm{~g}$. Con este dispositivo se podían realizar varios experimentos; el que interesaba para este trabajo era t-2: dos carritos se ponian en contacto con los muelles listos para soltarse. Cuando se quitaba la traba de seguridad, los dos carritos recibían un impulso y adquirian una velocidad igual, recorriendo prácticamente el mismo camino antes de detenerse por rozamiento.

\section{APÉNDICE C: EJEMPLOS DE CONFLICTOS}

\section{a) Conflicto local}

CE-- Porque cuando la lanzamos con menos fuerza, la bolita continúa junto a la otra (realiza c-2)...

Continúa si la masa (del proyectil) es mayor oigual. Si la masa es menor (realiza c-6), se detiene; también si es lanzada con poca fuerza.

E.- Déjame hacer ил pequeño truco (realiza c-5).

CE.- ;Ah! Ha vuelto (desilusionada)... Si pongo la misma energía en una pequeña o en una grande, ¿cuál tendrá más velocidad? La pequeña, ¿ $e h$ ?

E.- ¿Por qué esta pregunta?

CE.-No sé, cuánto mayor es la velocidad... Si ésta es menor y tiene mayor velocidad... Según la velocidad que le das, avanza o vuelve. Si haces así (c-6), se detiene. Si haces asi (c-2), van juntos (al blanco) con la misma fuerza... Estoy pensando si la velocidad influye... Estoy pensando si la masa influye en la velocidad.»

\section{b) Conflicto en paralelo}

La entrevistadora había pedido al estudiante que hiciera una previsión sobre un experimento imaginario de tipo c-2, pero con bolitas de plastilina. $\mathrm{FE}$ previó que después del choque se obtendría un movimiento oscilatorio de las dos bolitas unidas, causado probablemente, «por una fuerza elástica (entre las dos bolitas) que acompañaba el movimienton. La entrevistadora introdujo entonces un nuevo experimento imaginario, siempre de tipop-2, pero con bolitas de goma, pidiéndole que confrontara los dos casos. FE confirmó la diferencia: existía una «fuerza elástica» entre las bolitas de plastilina que explicaba el movimiento oscilatorio y una "fuerza anelástica» entre las bolitas de goma, que explicaba su rebote despues del choque. Sin embargo, quedaba una duda sobre cómo explicar el caso de c-8, con las bolitas de acero que rebotaban después del choque.

FE.-Usted dijo que hay dos maneras de lanzar las bolitas, ¿no es cierto?

E.-Sí.

FE. - Un manera es que la bolita $A$ se acerque a $B$ (muestra las dos bolitas acercándose una a la otra). Luego habrá una interacción entre ellas...y luego se alejarán. ¿Por qué ocurre así? ¿No podrian (las bolitas de acero) detenerse aquí en el punto de contacto entre las dos?

Para FE los dos modos de ianzamiento se caracterizaban por producir o no rebote: en cambio para la entrevistadora se caracterizaban por el deslizamiento o por el rodamiento.

E.- ¿Por qué vuelven? ¿Tu pregunta tiene que ver con el modo diferente de lanzarlas o tal vez no?

FE.- Exactamente. Debe tratarse de alguna cosa de anelastico o algo parecido, porque si cogiéramos estas dos de aquí (muestra las dos bolitas de plastilina), se unirian...

La entrevistadora no aprovechó la sugerencia de analogía (alguna cosa de «anelástico») entre las bolitas de goma y las de acero porque pensaba en el conflicto objetivo aparecido precedentemente (el movimiento oscilatorio con las dos bolitas 
de plastilina), por esto introdujo un choque frontal de tipo c-8 con bolitas de plastilina y le pidió nuevamente una confrontación con el correspondiente de tipo p-2. FE confirmó que en c-8 Ias bolitas de plastilina se habrían detenido, mientras en p-2 habrían continuando oscilando juntas.

E.- ¿Por qué allâ (en el péndulo) se mueven y aquí (en el carril) se detienen?

FE.- Porque... porque en el contacto, en la interacción aqui (señala las dos bolitas en el carriI)... sera que existe una fuerza elástica que impide el alejamiento (reciproco)?

La entrevistadora dttaba de si responder inmediatamente, cuando fue internumpida por el estudiante que sugirió una nueva hipótesis: Tal vez en el choque entre las bolitas de plastilina se podría observar un alejamiento recíproco, como en el caso de las bolitas de acero, si se modificara su masa y su velocidad. La entrevistadora, no teniendo clara la situación conflictiva del estudiante, aprovechó Ia idea para recapitular los diversos casos analizados anteriormente con las bolitas de acero, con la esperanza de resolver otro conflicto que había quedado abierto, la diferencia entre c-1 y c-2. Pero para FE el problema con las bolitas de acero exa diferente y mucho mas profundo, como teveló luego: ¿Por qué algunas veces las bolitas se detienen o continúan moviéndose en el mismo sentido del lanzamiento y otras veces rebotan?

\section{c) Conflicto en serie}

NA había hecho la previsión de que en c-5 el proyectil se detendría «transmitiendo toda su velocidad al blanco». Al observar varias veces el rebote del blanco después del choque dijo que el efecto era debido a la presencia de la acción y la reacción. La entrevistadora preguntó si no era posible explicarlo mediante la transmision de energía (ya usada anteriormente por el estudiante).

NA.- No, sería la transmisión de la fuerza... La fuerza que actúa sobre la bolita (blanco) es restituida... la reacción ha desplazado a la otra (el proyectil).

E.- Acción y reacción: ¿cuál es mayor?

NA.- Son iguales.

E.- ¿Por qué los efectos son entonces diferentes? (repite el experimento dos veces). ¿Cuál tiene mayor velocidad?

NA.- La mayor... la fuerza es masa por aceleración... si la masa es mayor y la aceleración constante, la fuerza es mayor.

E.- Pero ¿no hemos partido de fuerzas iguales?

NA.- La velocidad de la bolita pequeña es menor, porque la fuerza es igual y la masa es menor...

E. - ¿Estás pensando en la relación $F=m . a$ ?

NA.- $S i$.

E.- ¿(Tu razonamiento es que) la velocidad es menor y la masa es menor; por lo tanto, la fuerza es menor?

NA.- No, la fuerza es constante... No, la fuerza debe ser diferente, porque... la fuerza es constante y la aceleración de una es mayor que la de la otra... No, la velocidad de la (bolita) menor es mayor que la de la mayor...

E.- Pero, ¿no habías observado que la velocidad de la menor era menor?

NA.- No, la velocidad de la menor es mayor.
E.-Veámoslo nuevamente (repite dos veces el experimento.)

NA.- Entonces... no sé. Si la aceleración de la bolita mayor es mayor, entonces la fuerza que está en ella es mayor.

El diálogo continuó con NA afirmando haber aprendido en la escuela que acción y reacción eran iguales y que tal vez en este caso el sistema no era aislado y existían fuerzas externas, como el rozamiento y la resistencia del aire. La entrevistadora sugirió analizar el caso de p-4.

E.- ¿Qué sucederá si abandonamos la bolita menor desde una cierta altura?

NA.- Acción y reacción deben ser iguales; por lo tanto, las dos bolitas deben alcanzar la misma altura.

E.- ¿Aúnque las masas sean diferentes?

NA.- No, espera. Acción igual a reacción... entonces... la menor debe alcanzar una altura mayor.

E.-Veamos (realiza algunas veces el experimento). Probemos partiendo desde más alto, para ver mejor el efecto del primer choque (realiza nutevamente p-4).

NA.- La menor llegó más abajo... no sé.

E.- Para ti, ¿la menor debía llegar a mayor altura para estar de acuerdo con la igualdad de la acción y de la reacción?

NA.- Sí... ¿Por qué la menor fue más lenta? ¿La acción no es igual a la reacción? ... ¿La acción es igual a la reacción o no?

\section{d) Conflicto en espiral}

Después de haber explicado p-1 y p-5 como ejemplos de transferencia de energía, THA quedó muy sorprendida del rebote del proyectil en $\mathrm{p}-\phi$, buscando una posible explicación en la idea de la transferencia total en la dirección del movimiento, de la energía de la bolita media a la grande hasta el momento de su detención, y en cualquier mecanismo desconocido responsable del rebote del proyectil. Después de intentar aplicar el esquema de transferencia total también a p- 5 , en el choque entre una bolita y un blanco medio hasta modificar el resultado del experimento, debió reconocer su equivovación frente a una observación más atentz; como resultado, el modelo de transferencia total continuó siendo válido sólo para p-1 y el rebote del proyectil en p- 4 permaneciós sin explicación. El diálogo prosiguió desplazando la atención al problema de la clarificación de la diferencia entre c-1 y c-2, hasta llegar a un cierto momento en que la entrevistadora sugirió consiđerar las fuerzas durante el choque. Después de analizar varios casos se volvió al problema de $\mathrm{p}-4$, esta vez resuelto inmediatamente por THA mediante la idea de acción y reacción. Pero esta solución dio origen a un nuevo problema: el rol de la acción y reacción en los experimentos, incluso en aquéllos realizados con el carril. Se obtuvo un nuevo paso adelante cuando se reconoció que la reacción en c-1 era responsable de la detención del proyectil y el conflicto fue parcialmente resuelto; el diálogo se desplazó sobre el análisis de la fuerza durante el lanzamiento de un proyectil y la entrevistadora discutió detalladamente la reiación $\mathrm{f}=\mathrm{m}$. $a$ y el problema de la relación entre fuerza y movimiento. Finalmente retornando al análisis de los choques, el rebote del proyectil en p.4 se confirmó como efecto de la reacción, pero THA manifestó una duda sobre la validez de la misma explicación en el caso p-1 aunque el problema parecía estar tesuelto anteriormente. Desafortunadamente la entrevistadora no percibió la duda de la estudiante y consideró el problema como resuelto definitivamente, olvidando también de tratar explícitamente el caso de p-5. 